\title{
An application of the inequality for modified Poisson kernel
}

\author{
Gaixian Xue and Junfei Wang ${ }^{2^{*}}$
}

"Correspondence: qzifwang@qq.com

${ }^{2}$ Foundation Department, Wuhai Vocational and Technical College, Wuhai, 016000, China Full list of author information is available at the end of the article

\begin{abstract}
As an application of an inequality for modified Poisson kernel obtained by Qiao and Deng (Bull. Malays. Math. Sci. Soc. (2) 36(2):511-523, 2013), we give the generalized solution of the Dirichlet problem with arbitrary growth data.
\end{abstract}

Keywords: growth property; Dirichlet problem; modified Poisson kernel

\section{Introduction and results}

Let $\mathbf{R}^{n}(n \geq 2)$ be the $n$-dimensional Euclidean space. The boundary and the closure of a set $E$ in $\mathbf{R}^{n}$ are denoted by $\partial E$ and $\bar{E}$, respectively. The Euclidean distance of two points $P$ and $Q$ in $\mathbf{R}^{n}$ is denoted by $|P-Q|$. Especially, $|P|$ denotes the distance of two points $P$ and $O$ in $\mathbf{R}^{n}$, where $O$ is the origin in $\mathbf{R}^{n}$.

We introduce a system of spherical coordinates $(r, \Theta), \Theta=\left(\theta_{1}, \theta_{2}, \ldots, \theta_{n-1}\right)$, in $\mathbf{R}^{n}$ which are related to Cartesian coordinates $\left(x_{1}, x_{2}, \ldots, x_{n-1}, x_{n}\right)$ by $x_{n}=r \cos \theta_{1}$.

Let $B(P, r)$ denote the open ball with center at $P$ and radius $r(>0)$ in $\mathbf{R}^{n}$. The unit sphere and the upper half unit sphere in $\mathbf{R}^{n}$ are denoted by $\mathbf{S}^{n-1}$ and $\mathbf{S}_{+}^{n-1}$, respectively. The surface area $2 \pi^{n / 2}\{\Gamma(n / 2)\}^{-1}$ of $\mathbf{S}^{n-1}$ is denoted $w_{n}$. Let $\Omega \subset \mathbf{S}^{n-1}$, a point $(1, \Theta)$ and the set $\{\Theta ;(1, \Theta) \in \Omega\}$ are denoted $\Theta$ and $\Omega$, respectively. For two sets $\Lambda \subset \mathbf{R}_{+}$and $\Omega \subset \mathbf{S}^{n-1}$, we denote $\Lambda \times \Omega=\left\{(r, \Theta) \in \mathbf{R}^{n} ; r \in \Lambda,(1, \Theta) \in \Omega\right\}$, where $\mathbf{R}_{+}$is the set of all positive real numbers.

For the set $\Omega \subset \mathbf{S}^{n-1}$, we denote the set $\mathbf{R}_{+} \times \Omega$ in $\mathbf{R}^{n}$ by $C_{n}(\Omega)$, which is called a cone. For the set $I \subset \mathbf{R}$, the sets $I \times \Omega$ and $I \times \partial \Omega$ are denoted $C_{n}(\Omega ; I)$ and $S_{n}(\Omega ; I)$, respectively, where $\mathbf{R}$ is the set of all real numbers. Especially, the set $S_{n}\left(\Omega ; \mathbf{R}_{+}\right)$is denoted $S_{n}(\Omega)$.

Given a continuous function $f$ on $S_{n}(\Omega)$, we say that $h$ is a solution of the Dirichlet problem in $C_{n}(\Omega)$ with $f$, if $h$ is a harmonic function in $C_{n}(\Omega)$ and

$$
\lim _{P \rightarrow Q \in S_{n}(\Omega), P \in C_{n}(\Omega)} h(P)=f(Q) .
$$

Let $\Omega \subset \mathbf{S}^{n-1}$ and $\Delta^{*}$ be a Laplace-Beltrami on the unit sphere. Consider the Dirichlet problem (see, e.g. [2], p.41)

$$
\begin{aligned}
& \Delta^{*} \varphi(\Theta)+\lambda \varphi(\Theta)=0 \quad \text { in } \Omega, \\
& \varphi(\Theta)=0 \quad \text { in } \partial \Omega .
\end{aligned}
$$

(c) 2016 Xue and Wang. This article is distributed under the terms of the Creative Commons Attribution 4.0 International License (http://creativecommons.org/licenses/by/4.0/), which permits unrestricted use, distribution, and reproduction in any medium, provided you give appropriate credit to the original author(s) and the source, provide a link to the Creative Commons license, and indicate if changes were made. 
We denote the non-decreasing sequence of positive eigenvalues of it, repeating accordingly to their multiplicities, and the corresponding eigenfunctions are denoted, respectively, by $\left\{\lambda_{i}\right\}_{i=1}^{\infty}$ and $\left\{\varphi_{i}(\Theta)\right\}_{i=1}^{\infty}$. Especially, we denote the least positive eigenvalue of it $\lambda_{1}$ and the normalized positive eigenfunction to $\lambda_{1} \varphi_{1}(\Theta)$. In the sequel, for the sake of brevity, we shall write $\lambda$ and $\varphi$ instead of $\lambda_{1}$ and $\varphi_{1}$, respectively.

The set of sequential eigenfunctions corresponding to the same value of $\left\{\lambda_{i}\right\}_{i=1}^{\infty}$ in the sequence $\left\{\varphi_{i}(\Theta)\right\}_{i=1}^{\infty}$ makes an orthonormal basis for the eigenspace of the eigenvalue $\lambda_{i}$. Hence for each $\Omega \subset S^{n-1}$ there is a sequence $\left\{k_{j}\right\}$ of positive integers such that $k_{1}=1, \lambda_{k_{j}}<$ $\lambda_{k_{j+1}}, \lambda_{k_{j}}=\lambda_{k_{j+1}}=\lambda_{k_{j+2}}=\cdots=\lambda_{k_{j+1}-1}$ and $\left\{\varphi_{k_{j}}, \varphi_{k_{j+1}}, \ldots, \varphi_{k_{j+1}-1}\right\}$ is an orthonormal basis for the eigenspace of the eigenvalue $\left\{\lambda_{k_{j}}\right\}_{j=1}^{\infty}$. By $I_{\Omega}\left(k_{m}\right)$ we denote the set of all positive integers less than $\left\{k_{m}\right\}_{m=1}^{\infty}$. In spite of the fact

$$
I_{\Omega}\left(k_{1}\right)=\varnothing,
$$

the summation over $I_{\Omega}\left(k_{1}\right)$ of a function $S(k)$ of a variable $k$ will be used by promising

$$
\sum_{k \in I_{\Omega}\left(k_{1}\right)} S(k)=0
$$

If we denote the solutions of the equation

$$
t^{2}+(n-2) t-\lambda_{i}=0 \quad(i=1,2,3, \ldots)
$$

by $\aleph_{i}^{+}$and $\aleph_{i}^{-}$, then the functions

$$
r^{\aleph_{i}^{ \pm}} \varphi_{i}(\Theta) \quad(i=1,2,3, \ldots)
$$

are harmonic functions in $C_{n}(\Omega)$ and vanish on $S_{n}(\Omega)$.

Let $G_{\Omega}(P, Q)$ be the Green function of $C_{n}(\Omega)$ for any $P=(r, \Theta) \in C_{n}(\Omega)$ and any $Q=$ $(t, \Phi) \in C_{n}(\Omega)$. Then the Poisson kernel in $C_{n}(\Omega)$ can be defined by

$$
P I_{\Omega}(P, Q)=\frac{1}{c_{n}} \frac{\partial}{\partial n_{Q}} G_{\Omega}(P, Q)
$$

where $P \in C_{n}(\Omega), Q \in S_{n}(\Omega), \partial / \partial n_{Q}$ denotes the differentiation at $Q$ along the inward normal into $C_{n}(\Omega)$ and

$$
c_{n}= \begin{cases}2 \pi & \text { if } n=2 \\ (n-2) w_{n} & \text { if } n \geq 3 .\end{cases}
$$

Let $F(\Theta)$ be a function defined in $\Omega$. We denote $N_{i}(F)$ by

$$
\int_{\Omega} F(\Theta) \varphi_{i}(\Theta) d \Omega,
$$

when it exists. 
For any two points $P=(r, \Theta)$ and $Q=(t, \Phi)$ in $C_{n}(\Omega)$ and $S_{n}(\Omega)$, respectively, we define

$$
\widetilde{K}_{\Omega}^{m}(P, Q)= \begin{cases}0 & \text { if } 0<t<1 \\ K_{\Omega}^{m}(P, Q) & \text { if } 1 \leq t<\infty\end{cases}
$$

where $m$ is a non-negative integer and

$$
K_{\Omega}^{m}(P, Q)=\sum_{i \in I_{k_{m+1}}} 2^{\aleph_{i}^{+}+n-1} N_{i}\left(P I_{\Omega}((1, \Theta),(2, \Phi))\right) r^{\aleph_{i}^{+}} t^{-\aleph_{i}^{+}-n+1} \varphi_{i}(\Theta)
$$

To obtain the solution of the Dirichlet problem in a cone, as in $[1,3,4]$, we use the modified Poisson kernel defined by

$$
P I_{\Omega}^{m}(P, Q)=P I_{\Omega}(P, Q)-\widetilde{K}_{\Omega}^{m}(P, Q),
$$

where $P \in C_{n}(\Omega)$ and $Q \in S_{n}(\Omega)$, which has the following estimates (see [1]):

$$
\left|P I_{\Omega}(P, Q)-K_{\Omega}^{m}(P, Q)\right| \leq M(2 r)^{\aleph_{k_{m+1}}^{+}} t^{-\aleph_{k_{m+1}^{+}}^{-n+1}}
$$

for any $P=(r, \Theta) \in C_{n}(\Omega)$ and any $Q=(t, \Phi) \in S_{n}(\Omega)$ satisfying $0<\frac{r}{t}<\frac{1}{2}$, where $M$ is a constant independent of $P, Q$, and $m$. For the construction and applications of a modified Green function in a half space, we refer the reader to the paper by Qiao (see [5]).

Write

$$
U_{\Omega}^{m}[f](P)=\int_{S_{n}(\Omega)} P I_{\Omega}^{m}(P, Q) f(Q) d \sigma_{Q}
$$

where $f(Q)$ is a continuous function on $\partial C_{n}(\Omega)$ and $d \sigma_{Q}$ the $(n-1)$-dimensional volume elements induced by the Euclidean metric on $\partial C_{n}(\Omega)$.

Recently, Qiao and Deng (cf. [1]) gave the solution of the Dirichlet problem in a cone. Applications of modified Poisson kernel with respect to the Schrödinger operator, we refer the reader to the papers by Huang and Ychussie (see [6]) and Li and Ychussie (see [7]).

Theorem A If $\Omega+\aleph^{+}-1>0, \Omega-n+1 \leq \aleph_{k_{m+1}^{+}}<\Omega-n+2$ and $f(Q)(Q=(t, \Phi))$ is a continuous function on $\partial C_{n}(\Omega)$ satisfying

$$
\int_{S_{n}(\Omega)} \frac{|f(Q)|}{1+t^{\Omega}} d \sigma_{Q}<\infty
$$

then the function $U_{\Omega}^{m}[f](P)$ is a solution of the Dirichlet problem in $C_{n}(\Omega)$ with $f$ and

$$
\lim _{r \rightarrow \infty, P=(r, \Theta) \in C_{n}(\Omega)} r^{n-\Omega-1} \varphi^{n-1}(\Theta) U_{\Omega}^{m}[f](P)=0
$$

Furthermore, Qiao and Deng (cf. [4]) supplemented the above result and proved the following. 
Theorem B Let $0<p<\infty, \gamma>\left(-\aleph^{+}-n+2\right) p+n-1$ and

$$
\frac{\gamma-n+1}{p}<\aleph_{k_{m+1}}^{+}<\frac{\gamma-n+1}{p}+1
$$

Iff $(Q)(Q=(t, \Phi))$ is a continuous function on $S_{n}(\Omega)$ satisfying

$$
\int_{S_{n}(\Omega)} \frac{|f(Q)|^{p}}{1+t^{\gamma}} d \sigma_{Q}<\infty
$$

then the function $U_{\Omega}^{m}[f](P)$ satisfies

$$
\lim _{r \rightarrow \infty, P=(r, \Theta) \in C_{n}(\Omega)} r^{\frac{n-\gamma-1}{p}} \varphi^{n-1}(\Theta) U_{\Omega}^{m}[f](P)=0 .
$$

It is natural to ask if the continuous function $u$ satisfying (2) and (3) can be replaced by arbitrary continuous function? In this paper, we shall give an affirmative answer to this question. To do this, we first construct a modified Poisson kernel. Let $\phi(l)$ be a positive function of $l \geq 1$ satisfying

$$
2^{\aleph^{+}} \phi(1)=1
$$

Denote the set

$$
\left\{l \geq 1 ;-\aleph_{k_{i}}^{+} \log 2=\log \left(l^{n-1} \phi(l)\right)\right\}
$$

by $\pi_{\Omega}(\phi, i)$. Then $1 \in \pi_{\Omega}(\phi, i)$. When there is an integer $N$ such that $\pi_{\Omega}(\phi, N) \neq \Phi$ and $\pi_{\Omega}(\phi, N+1)=\Phi$, denote

$$
J_{\Omega}(\phi)=\{i ; 1 \leq i \leq N\}
$$

of integers. Otherwise, denote the set of all positive integers by $J_{\Omega}(\phi)$. Let $l(i)=l_{\Omega}(\phi, i+$ 1) be the minimum elements $l$ in $\pi_{\Omega}(\phi, i)$ for each $i \in J_{\Omega}(\phi)$. In the former case, we put $l(N+1)=\infty$. Then $l(1)=1$. The kernel function $\widetilde{K}_{\Omega}^{\phi}(P, Q)$ is defined by

$$
\widetilde{K}_{\Omega}^{\phi}(P, Q)= \begin{cases}0 & \text { if } 0<t<1, \\ K_{\Omega}^{i}(P, Q) & \text { if } l(i) \leq t<l(i+2) \text { and } i \in J_{\Omega}(\phi)\end{cases}
$$

where $P \in C_{n}(\Omega)$ and $Q=(t, \Phi) \in S_{n}(\Omega)$.

The generalized Poisson kernel $P_{\Omega}^{\phi}(P, Q)$ is defined by

$$
P I_{\Omega}^{\phi}(P, Q)=P I_{\Omega}(P, Q)-\widetilde{K}_{\Omega}^{\phi}(P, Q)
$$

where $P \in C_{n}(\Omega)$ and $Q \in S_{n}(\Omega)$.

As an application of the inequality (1) and the generalized Poisson kernel $P I_{\Omega}^{\phi}(P, Q)$, we have the following. 
Theorem $\operatorname{Let} g(Q)$ be a continuous function on $S_{n}(\Omega)$. Then there is a positive continuous function $\phi_{g}(l)$ of $l \geq 0$ depending on $g$ such that

$$
H_{\Omega}^{\phi_{g}}(P)=\int_{S_{n}(\Omega)} P I_{\Omega}^{\phi_{g}}(P, Q) g(Q) d \sigma_{Q}
$$

is a solution of the Dirichlet problem in $C_{n}(\Omega)$ with $g$.

\section{Lemmas}

Lemma 1 Let $\phi(l)$ be a positive continuous function of $l \geq 1$ satisfying

$$
\phi(1)=2^{-\aleph^{+}} .
$$

Then

$$
\left|P I_{\Omega}(P, Q)-\widetilde{K}_{\Omega}^{\phi}(P, Q)\right| \leq M \phi(l)
$$

for any $P=(r, \Theta) \in C_{n}(\Omega)$ and any $Q=(t, \Phi) \in S_{n}(\Omega)$ satisfying

$$
t>\max \{1,4 r\} .
$$

Proof We can choose two points $P=(r, \Theta) \in C_{n}(\Omega)$ and $Q=(t, \Phi) \in S_{n}(\Omega)$, which satisfies (4). Moreover, we also can choose an integer $i=i(P, Q) \in J_{\Omega}(\phi)$ such that

$$
l(i-1) \leq t<l(i)
$$

Then

$$
\widetilde{K}_{\Omega}^{\phi}(P, Q)=K_{\Omega}^{i-1}(P, Q)
$$

Hence we have from (1), (4), and (5) that

$$
\left|P I_{\Omega}(P, Q)-\widetilde{K}_{\Omega}^{\phi}(P, Q)\right| \leq M 2^{-\aleph_{k_{i}}^{+}} \leq M \phi(l)
$$

which is the conclusion.

Lemma 2 (See [4]) Let $g(Q)$ be a continuous function on $\partial C_{n}(\Omega)$ and $V(P, Q)$ be a locally integrable function on $\partial C_{n}(\Omega)$ for any fixed $P \in C_{n}(\Omega)$, where $Q \in \partial C_{n}(\Omega)$. Define

$$
W(P, Q)=P I_{\Omega}(P, Q)-V(P, Q)
$$

for any $P \in C_{n}(\Omega)$ and any $Q \in \partial C_{n}(\Omega)$.

Suppose that the following two conditions are satisfied:

(I) For any $Q^{\prime} \in \partial C_{n}(\Omega)$ and any $\epsilon>0$, there exists a neighborhood $B\left(Q^{\prime}\right)$ of $Q^{\prime}$ such that

$$
\int_{S_{n}(\Omega ;[R, \infty))}|W(P, Q)||u(Q)| d \sigma_{Q}<\epsilon
$$

for any $P=(r, \Theta) \in C_{n}(\Omega) \cap B\left(Q^{\prime}\right)$, where $R$ is a positive real number. 
(II) For any $Q^{\prime} \in \partial C_{n}(\Omega)$, we have

$$
\underset{P \rightarrow Q^{\prime}, P \in C_{n}(\Omega)}{\limsup } \int_{S_{n}(\Omega ;(0, R))}|V(P, Q)||u(Q)| d \sigma_{Q}=0
$$

for any positive real number $R$.

Then

$$
\limsup _{P \rightarrow Q^{\prime}, P \in C_{n}(\Omega)} \int_{S_{n}(\Omega)} W(P, Q) u\left(Q^{\prime}\right) d \sigma_{Q} \leq u(Q)
$$

for any $Q^{\prime} \in \partial C_{n}(\Omega)$.

\section{Proof of Theorem}

Take a positive continuous function $\phi(l)(l \geq 1)$ such that

$$
\phi(1) 2^{\aleph^{+}}=1
$$

and

$$
\phi(l) \int_{\partial \Omega}|g(l, \Phi)| d \sigma_{\Phi} \leq \frac{L}{l^{n}}
$$

for $l>1$, where

$$
L=2^{-\aleph^{+}} \int_{\partial \Omega}|g(1, \Phi)| d \sigma_{\Phi}
$$

For any fixed $P=(r, \Theta) \in C_{n}(\Omega)$, we can choose a number $R$ satisfying $R>\max \{1,4 r\}$. Then we see from Lemma 1 that

$$
\begin{aligned}
& \int_{S_{n}(\Omega ;(R, \infty))}\left|P I_{\Omega}^{\phi_{g}}(P, Q)\right||g(Q)| d \sigma_{Q} \\
& \quad \leq M \int_{R}^{\infty}\left(\int_{\partial \Omega}|g(1, \Phi)| d \sigma_{\Phi}\right) \phi(l) l^{n-2} d l \\
& \leq M L \int_{R}^{\infty} l^{-2} d l \\
& \leq \infty .
\end{aligned}
$$

Obviously, we have

$$
\int_{S_{n}(\Omega ;(0, R))}\left|P I_{\Omega}^{\phi_{g}}(P, Q)\right||g(Q)| d \sigma_{Q}<\infty
$$

which gives

$$
\int_{S_{n}(\Omega)}\left|P I_{\Omega}^{\phi_{g}}(P, Q)\right||g(Q)| d \sigma_{Q}<\infty
$$

To see that $H_{\Omega}^{\phi_{g}}(P)$ is a harmonic function in $C_{n}(\Omega)$, we remark that $H_{\Omega}^{\phi_{g}}(P)$ satisfies the locally mean-valued property by Fubini's theorem. 
Finally we shall show that

$$
\lim _{P \in C_{n}(\Omega), P \rightarrow Q^{\prime}} H_{\Omega}^{\phi_{g}}(P)=g\left(Q^{\prime}\right)
$$

for any $Q^{\prime}=\left(t^{\prime}, \Phi^{\prime}\right) \in \partial C_{n}(\Omega)$. Set

$$
V(P, Q)=\widetilde{K}_{\Omega}^{\phi_{g}}(P, Q)
$$

in Lemma 2, which is locally integrable on $S_{n}(\Omega)$ for any fixed $P \in C_{n}(\Omega)$. Then we apply Lemma 2 to $g(Q)$ and $-g(Q)$.

For any $\epsilon>0$ and a positive number $\delta$, by (9) we can choose a number $R\left(>\max \left\{1,2\left(t^{\prime}+\right.\right.\right.$ $\delta)\}$ ) such that (6) holds, where $P \in C_{n}(\Omega) \cap B\left(Q^{\prime}, \delta\right)$.

Since

$$
\lim _{\Theta \rightarrow \Phi^{\prime}} \varphi_{i}(\Theta)=0 \quad(i=1,2,3 \ldots)
$$

as $P=(r, \Theta) \rightarrow Q^{\prime}=\left(t^{\prime}, \Phi^{\prime}\right) \in S_{n}(\Omega)$, we have

$$
\lim _{P \in C_{n}(\Omega), P \rightarrow Q^{\prime}} \widetilde{K}_{\Omega}^{\phi_{g}}(P, Q)=0
$$

where $Q \in S_{n}(\Omega)$ and $Q^{\prime} \in S_{n}(\Omega)$. Then (7) holds.

Thus we complete the proof of Theorem.

\section{Competing interests}

The authors declare that they have no competing interests.

\section{Authors' contributions}

All authors contributed equally to the manuscript and read and approved the final manuscript.

\section{Author details}

'School of Mathematics and Information Science, Henan University of Economics and Law, Zhengzhou, 450002, China.

${ }^{2}$ Foundation Department, Wuhai Vocational and Technical College, Wuhai, 016000, China.

\section{Acknowledgements}

The authors are very thankful to the anonymous referees for their valuable comments and constructive suggestions.

Received: 14 September 2015 Accepted: 4 January 2016 Published online: 22 January 2016

\section{References}

1. Qiao, L, Deng, GT: Growth property and integral representation of harmonic functions in a cone. Bull. Malays. Math. Soc. 36(2), 511-523 (2013)

2. Rosenblum, G, Solomyak, M, Shubin, M: Spectral Theory of Differential Operators. VINITI, Moscow (1989)

3. Qiao, L: Integral representations for harmonic functions of infinite order in a cone. Results Math. 61(1-2), 63-74 (2012)

4. Qiao, L: Growth of certain harmonic functions in an $n$-dimensional cone. Front. Math. China 8(4), 891-905 (2013)

5. Qiao, L: Modified Poisson integral and Green potential on a half-space. Abstr. Appl. Anal., 2012, Article ID 765965 (2012)

6. Huang, J, Ychussie, B: The modification of Poisson-Sch integral on cones and its applications. Filomat (to appear)

7. Li, Z, Ychussie, B: Sharp geometrical properties of a-rarefied sets via fixed point index for the Schrödinger operator equations. Fixed Point Theory Appl. 2015, 89 (2015) 